Research Article

\title{
Overexpression of salt-induced protein (salT) delays leaf senescence in rice
}

\author{
Keming Zhu ${ }^{1}$ D, Huimin $\mathrm{Tao}^{1}$, Shuo $\mathrm{Xu}^{1}$, Kaixia $\mathrm{Li}^{1}$, Sundus Zafar ${ }^{1}$, Wei Cao ${ }^{1}$ and Yanhua Yang ${ }^{1}$ \\ ${ }^{1}$ Institute of Life Sciences, Jiangsu University, Zhenjiang, Jiangsu, People's Republic of China.
}

\begin{abstract}
Senescence, a highly programmed process, largely determines yield and quality of crops. However, knowledge about the onset and progression of leaf senescence in crop plants is still limited. Here, we report that salt-induced protein (salT), a new gene, may be involved in leaf senescence. Overexpressing salT could prolong the duration of leaves with higher concentrations of chlorophyll compared with the wild type. Moreover, overexpression of salT could delay the senescence of rice leaves though the inhibition of senescence associated genes (SAGs). Overall, the characterization of salT suggested that it is a new gene affecting the leaf senescence induced by natural and dark conditions.
\end{abstract}

Keywords: Leaf senescence, salt-induced protein (salT), rice, stay-green.

Received: December 05, 2017; Accepted: April 27,2018

\section{Introduction}

Senescence is the final phase of plant development, in which the plant goes through a series of programmed cell death (PCD) processes (Cao et al., 2003) \{Cao, $2003 \# 1\}$. This process combines chlorophyll degradation with carotenoid retention or anthocyanin accumulation (Park et al., 2007) so the leaves of plant generally change from green to yellow during senescence. It has been noted for long that leaf senescence is a major determinant of yield for many crops (Richards, 2000; Long et al., 2006; Yang and Zhang 2006). Delaying leaf senescence has usually been considered to be associated with the retention of the high photosynthetic capacity and yield increment (Thomas and Howarth, 2000; Masclaux-Daubresse and Chardon, 2011; Wu et al., 2016).

Rice (Oryza sativa L.) is one of the most important crops in the world and provides nearly half of the calories consumed by humankind (Zuo and Li, 2014). The leaf is the most important source organ for rice, with $60-80 \%$ of the nutrients required for grain filling after heading provided by leaf photosynthesis (Lu et al., 1988). Early-senescence of leaves will seriously reduce rice yield, disrupt the filling dynamics, and reduce grain quality (Zhao et al., 2014). The production can be increased by $2 \%$ with delaying rice senescence by one day (Liang et al., 2014). Therefore, finding an economic and effective method to delay leaf senescence in rice production can greatly improve the yield of rice.

During leaf senescence, the expression of senescence associated genes (SAGs) is up-regulated, which is a hall-

Send correspondence to Keming Zhu. Institute of Life Sciences, Jiangsu University, Xuefu Road, 301, Zhenjiang 212013, Jiangsu, People's Republic of China. E-mail: uegzkg@ sina.com.cn mark of leaf senescence (Kajimura et al., 2010). More than a thousand SAGs have been isolated, including transcription factors, signal transduction components, proteases, metabolic enzymes and various transporters of nutrients (Watanabe and Imaseki, 1982; John et al., 1997; Lee et al., 2001; Buchanan-Wollaston et al., 2003; Gregersen and Holm, 2007, Liu et al., 2008; Hayashi et al., 2015; Wu et al., 2016). Some important genes have been cloned and functionally characterized. STAY-GREEN $(S G R)$ is an important member of the metabolic pathway of chlorophyll degradation, which encodes a chloroplast transit peptide and regulates chlorophyll degradation by inducing lightharvesting proteins of photosystem II (LHCPII) disassembly through direct interaction (Jiang et al., 2007; Hörtensteiner; 2009). RED CHLOROPHYLL CATABOLITE REDUCTASE 1 (RCCRI) encodes a red chlorophyll catabolite reductase, plays a key role in the chlorophyll degradation pathway, and strongly participates in senescence (Tang et al., 2011). NON-YELLOW COLORING 1 (NYCl) encodes the chlorophyll b oxidation-reduction enzyme, and chlorophyll $\mathrm{b}$ cannot be degraded in the nycl mutant because of abnormal binding of light-harvesting chlorophyll and carotene (Kusaba et al., 2007). NONYELLOW COLORING 3 (NYC3) encodes a plastid-targeted $\alpha / \beta$ folding proteolytic enzyme that affects chloroplast structure, and the nyc 3 mutant exhibits a senescence phenotype (Morita et al., 2009). OsNAP encodes a transcription factor with a crucial role in regulating the senescence process, as demonstrated by the delayed senescence phenotype of Osnap mutants (Liang et al., 2014). In general, the onset and progression of leaf senescence is influenced by a number of endogenous and external factors. The mechanism of 
the onset of leaf senescence, especially the mechanism of delayed senescence in rice, is still largely unknown.

Here, we investigated the role of the salt-induced protein (salT) gene in rice. In our previous studies, we found OsISP specifically expressed in indica rice and the protein was identified as a salt-induced protein (salT) (Zhu et al., 2014). However, salT function is still not clear in rice. Here, we found that overexpression of salT in rice could delay the senescence of leaf by inhibiting the expression of SAGs and CDGs.

\section{Material and Methods}

\section{Plant materials and growth conditions}

All rice lines used in the study were derived from the japonica cultivar Nipponbare. Rice plants were cultivated in the experiment field at Jiangsu University, Zhenjiang, Jiangsu, during the natural growing season except where specifically indicated. For hydroponic culture, rice seedlings were grown in a constant-temperature incubator with light/dark of $16 / 8 \mathrm{~h}$ and $30-28{ }^{\circ} \mathrm{C}$, with approximately 200 $\mu \mathrm{mol}$ photons $/ \mathrm{m}^{2} / \mathrm{s}$ photon-density and $70 \%$ humidity. For dark incubation, the third fully expanded leaves of each genotype were excised and incubated under continuous darkness at $28^{\circ} \mathrm{C}$. All experiments were carried out using the same location of the leaves. The samples were collected with a sharp scalpel to minimize the impact of wounding, immediately frozen in liquid nitrogen, and stored at $-72{ }^{\circ} \mathrm{C}$ until needed for RNA isolation and chlorophyll extraction. All experiments were repeated three times independently.

\section{Vector construction and plant transformation}

Total RNA from rice leaf tissues (Zhonghua11) was extracted using TRIzol reagent (Invitrogen, Carlsbad, CA, USA). After removal of genomic DNA contamination by DNase I (TaKaRa, Dalian, China), 200?ng of poly(A)+ mRNA was converted into cDNA by MMLV Reverse Transcriptase (Vazyme, Nanjin, China). The cDNA template was subsequently used for PCR analysis. For salT (Os01g0348900), a full-length cDNA was obtained using the primers 5'-ATGACGCTGGTGAAGATTGG-3' and 5'-TCAAGGGTGGACGTAGATGC-3'.

To construct a vector for the constitutive expression of salT, a 438 bp full-length salT cDNA was PCR amplified from its cDNA clone with the primers 5'AAGTCGACATGACGCTGGTGAAGATTGG-3' and 5'-AACTGCAGTCAAGGGTGGACGTAGATGC-3', and inserted into the Sal I/ Pst I site of pCAMBIA1300Actin1-ocs, creating an overexpression vector, salT-OE. The inserted sequences were confirmed by restriction enzyme digestion and sequencing. To construct the salT RNAi vector, part of the salT cDNA was PCR amplified from its cDNA clone with the primers 5'-AAGGATCCATGACGCTGGT GAAGATTGG-3' and 5'-AAGTCGACATGGGTTCCAG AAATCTCCTT-3' and inserted into the BamH I/Sal I and
Xho I / Bgl II sites of the pUCCRNAi vector. Then, the pUCCRNAi vector was cut with Pst I and inserted into the Pst I site of pCAMBIA1300-Actin1-ocs, creating a salT RNAi vector, salT-RNAi. The inserted sequences were confirmed by restriction enzyme digestion and sequencing. The two binary plasmids were introduced into Agrobacterium tumefaciens EHA105 by electroporation and transformed into rice according to a published method (Hiei et al., 1994; Jeon et al., 2000).

\section{DNA, RNA extraction, and qRT-PCR}

Genomic DNA was extracted from rice leaves using the CTAB method and total RNA was extracted using the TRIzol reagent (Invitrogen). RNA was reversely transcribed from $3 \mu \mathrm{g}$ of total RNA with the M-MLV reverse transcriptase (Vazyme) according to the manufacturer's instructions. The qRT-PCR asays were carried out in a total volume of $20 \mu \mathrm{L}$, each containing $2 \mu \mathrm{L}$ of cDNA (200 ng), $10 \mu \mathrm{L}$ of SYBR Green Master Mix (Vazyme), $0.4 \mu \mathrm{L}$ of 50ROX Reference Dye I, $0.4 \mu \mathrm{L}$ of primers $(10 \mu \mathrm{M})$ and $7.2 \mu \mathrm{L}$ of $\mathrm{H}_{2} \mathrm{O}$. Cycling conditions included a hot start (5 min at $95^{\circ} \mathrm{C}$ ), followed by 40 cycles of $95^{\circ} \mathrm{C}$ for $10 \mathrm{~s}$, and $60{ }^{\circ} \mathrm{C}$ for $30 \mathrm{~s}$, and finished with $95^{\circ} \mathrm{C}$ for $15 \mathrm{~s}, 60^{\circ} \mathrm{C}$ for 60 $\mathrm{s}$, and $95^{\circ} \mathrm{C}$ for $15 \mathrm{~s}$. Amplification was performed using an ABI7300 PCR thermocycler (Applied Biosystems, USA). Rice Actin1 was chosen as a control to normalize all data. For analysis, a threshold was set for the change in fluorescence at a point in the linear PCR amplification phase. Melt curve analysis was performed to ensure a single product species. All experiments were done in duplicate for both target gene and internal control and were repeated three times independently. For the $\mathrm{CT}$ calculation to be valid, the amplification efficiencies of the target and reference must be approximately equal. The relative expression levels of genes were calculated using the $2^{-\ddot{A}} \Delta \mathrm{Ct}$ method (He et al., 2016; Tan et al., 2015). The statistical significance was analyzed by Student's $t$-test. The primer sequences that were used are listed in Table S1. PCR products were separated by electrophoresis on $1.0 \%(\mathrm{w} / \mathrm{v})$ agarose gels.

\section{Chlorophyll measurement}

Fresh rice leaves $(20 \mathrm{mg})$ were chilled with liquid nitrogen and ground to a powder. Chlorophyll was extracted with $80 \%$ acetone until the residue turned to white color, and diluted to a final volume of $25 \mathrm{~mL}$. Chlorophyll was determined by measuring absorbance at 663 and $645 \mathrm{~nm}$ using a W14546 spectrophotometer (Xin Mao Instrument, Shanghai, China). The chlorophyll content was calculated according to the formula $(\mathrm{Ca}=12.7$ OD663 - 2.69 OD645, $\mathrm{Cb}=22.9$ OD645 -4.68 OD663, $\mathrm{Ct}=8.02$ OD663 +20.21 OD645). Three seedlings of each sample were used in the chlorophyll assay. Statistical significance was analyzed by Student's $t$-test. Values are reported as mean \pm standard deviation of 10 measurements. 


\section{Results \\ Overexpressing salT delayed the senescence of rice leaves}

The OsISP protein was a protein marker for indica rice varieties, and was identified as salt-induced protein (salT), however the function of salT is still not clear in rice (Zhu et al., 2014). To further investigate its function, we constructed salT-overexpression (salT-OE) and salT-RNAi vectors, and generated transgenic plants by Agrobacterium mediated transformation (Hiei et al., 1994; Jeon et al., 2000). A total of eight positive salT-OE transgenic plants and 16 positive salT-RNAi transgenic plants were obtained. All eight salT-OE transgenic plants $\left(\mathrm{T}_{0}\right)$ maintained a stay-green phenotype in the mature stage (Figure 1A,B), but the phenotype of salT-RNAi transgenic rice was similar to the wild type (data not shown). Thus, we used the salT-OE transgenic rice lines for our study.
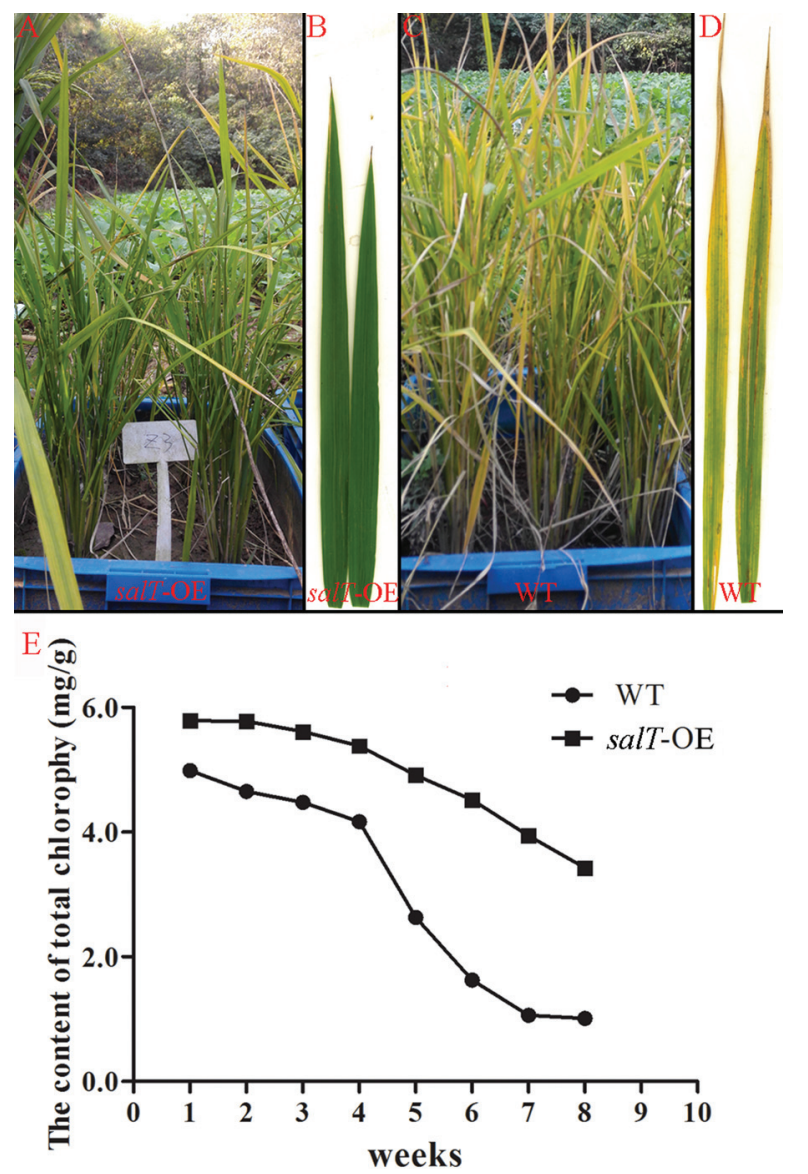

Figure 1 - Phenotype of wild-type and $\mathrm{T}_{0}$ transgenic plants carrying salT overexpression constructs (salT-OE) in the mature stage. (A) Phenotypes of the $\mathrm{T}_{0}$ salT-OE plants in the mature stage. (B) Flag leaf of the salT-OE plants keeps green in the mature stage. (C) Phenotypes of the wild type in the mature stage. (D) Flag leaf of the wild type turned yellow in the mature stage. (E) Chlorophyll contents of leaves of age-matched wild-type and salT-OE plants.
Total DNA was extracted from $\mathrm{T}_{0}$ transgenic rice plant and wild type leaves. In all $\mathrm{T}_{0}$ transgenic rice plants a $220 \mathrm{bp}$ fragment could be identified with hygromycin (hpt) gene-specific primers, which was similar to the positive control. However, no PCR products were detected in the wild type plants (Figure 2A). Moreover, we tested hygromycin resistance in excised leaves with hygromycin detection solution. All excised leaves from salT-OE transgenic plants stayed green and showed resistance to hygromycin while the leaves from untransformed control plants turned brown and died, indicating no resistance to hygromycin (Figure 2B). Furthermore, we extracted RNA from a salTOE transgenic plant, and the qRT-PCR results showed that the salT gene expression in salT-OE transgenic plant was about two times higher than that in wild type (Figure 2C). These results indicate that we successfully constructed salT-OE transgenic plants.

Phenotypic analysis of salT-OE transgenic plants was carried out. As shown in Figure 1, at the mature stage of rice, the wild type leaves turned yellow (Figure 1C and D) while the leaves of salT-OE rice had a stay-green phenotype (Figure 1A,B). We also quantified the chlorophyll content of flag leaves from the first week to the eighth week of the filling stage. During this period, the chlorophyll content of the leaves decreased gradually, but in salT-OE rice it decreased much slower than in wild type (Figure 1E). These results showed that overexpression of the salT gene in rice could delay the senescence of leaves.

\section{salT inhibits the expression of senescence associated genes}

The expression of SAGs are up-regulated during leaf senescence (Kajimura et al., 2010). Thus, we used quantita-

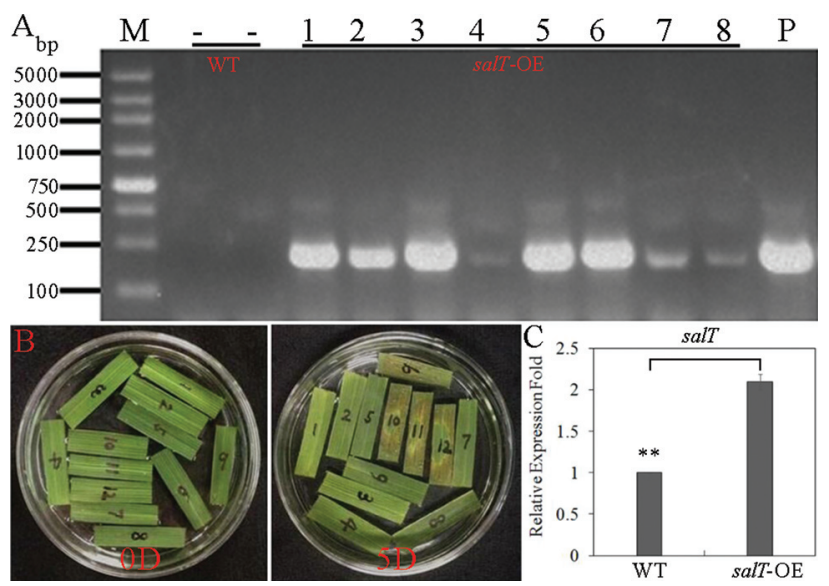

Figure 2 - Analysis of the salT-OE transgenic plants. (A) PCR analysis of hyg gene in the $\mathrm{T}_{0}$ salT-OE transgenic plants. M: DL2000 plus DNA maker; -: untransformed wild-type plants; 1-8: $\mathrm{T}_{0}$ salT-OE transgenic plants; P: pCAMBIA1300-Actin1-ocs plasmid. (B) Leaf assay of hygromycin resistance in transgenic rice plants. 1-8: $\mathrm{T}_{0}$ salT-OE transgenic plants; 9-12: untransformed wild type plants. (C) Transcript levels analysis the expression of salT gene between wild type and salT-OE transgenic plant. ${ }^{*} p<0.05,{ }^{*} p<0.01,{ }^{*} p<0.001$. Student's $t$-test was used to generate $p$-value. 
tive real-time PCR to detect two SAGs, OsNAP and OSCATB, in the flag leaves from wild type and salT-OE rice during the filling stage. As shown in Figure 3A, during the first week of the filling stage, the two genes were barely expressed in flag leaves. In the eighth week of the filling stage, the expression level of these two genes increased rapidly in the wild type, but not in the salT-OE plants. This result indicated that salT could inhibit leaf senescence by inhibiting the expression of SAGs.

Chlorophyll plays a central role in photosynthesis, and its degradation is an important phenomenon in leaf senescence (Morita et al., 2009). Therefore, we also quantified the expression levels of four chlorophyll degradationrelated genes (CDGs), OsSGR, OsNYC1, OsNYC3, and OsRCCR1, by real-time PCR during the filling stage. Our results showed that the expression of the four genes had almost no difference between wild type and salT-OE plants in the first week of the filling stage. At the eighth week of the filling stage, the expression level of these genes increased sharply in wild type. In salT-OE plants, the expression levels of the four genes also increased, but they were lower than in wild type (Figure 3B). This results suggests that overexpression of salT could keep leaves green through inhibiting chlorophyll degradation.
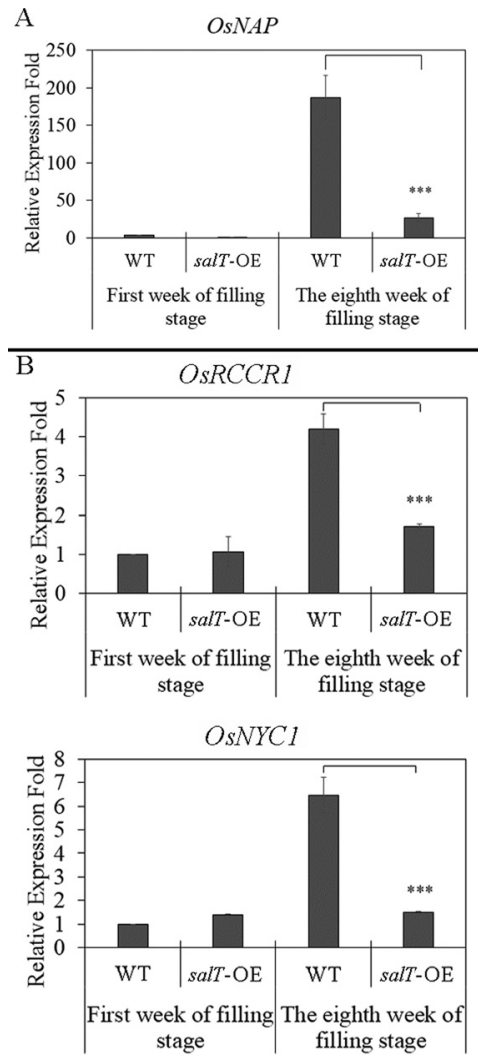

\section{Responses of salT to the dark treatment}

Dark treatment is the simplest and most efficient method to induce leaf senescence. While the detached leaves of Nipponbare (wild type) turned yellow 3 days after dark incubation (DAD), salT-OE retained the greenness at 5 DAD (Figure 4A). The chlorophyll content of rice seeding was examined during dark incubation. Initial chlorophyll contents were similar between wild type and salT-OE, but a prominent decrease was observed at 7 DAD in wild type (Figure 4B). The qRT-PCR results showed that two CDGs, $O s S G R$, and $O s R C C R$, had lower expression in salT-OE than in wild type plants after 7 DAD (Figure 4C). These observations indicate that salT-OE plants retain more chlorophyll than the wild type during senescence through inhibiting the expression of CDGs.

\section{Discussion}

Leaf senescence, the final stage of leaf development, occurs autonomously in an age-dependent manner and leads to the death of a cell or an organ. The aging process that leads to senescence and limitation of life is a common biological phenomenon in most organisms (Woo et al., 2001). Up to now, it is clear that leaf senescence is con-
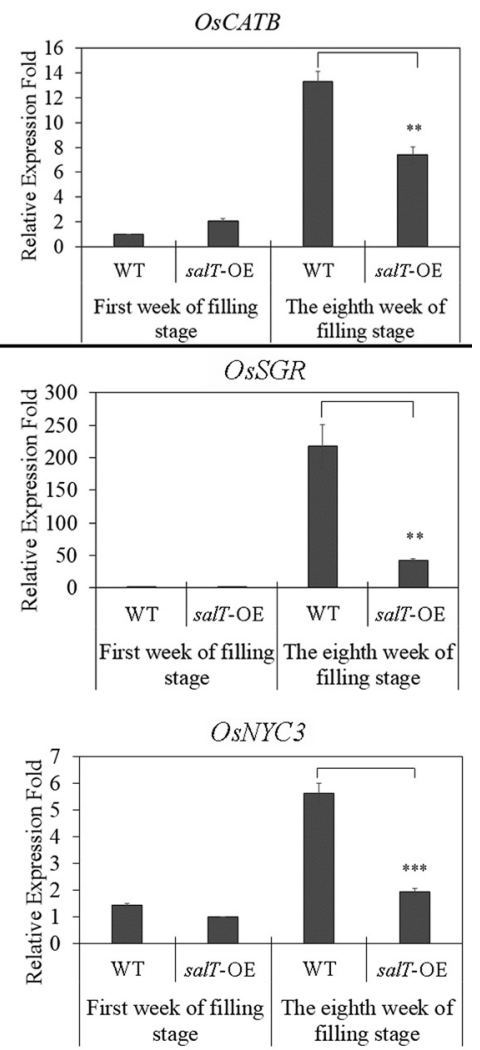

Figure 3 - Overexpression of salT inhibits the expression of senescence genes. (A) Relative transcript levels of senescence-associated genes in wild type and salT-OE plants during the filling stage. (B) Relative transcript levels of chlorophyll degradation-related genes (OsRCCR1, OsSGR, OsNYC1 and OsNYC3) of age-matched wild type leaves and salT-OE plants. Overexpression of salT gene delays dark-induced leaf senescence. Transcript levels are expressed relative to rice Actin 1 in each sample, and values are reported as mean $\pm \operatorname{SD}(\mathrm{n}=3)$. ${ }^{*} p<0.05,{ }^{*} p<0.01,{ }^{*} p<0.001$. Student's $t$-test was used to generate $p$-value. 
trolled by genes, and many leaf senescence genes in Arabidopsis, rice, maize, and barley have been cloned (Schippers, 2015; Raines et al., 2016; Sakuraba et al., 2016; Deng et al., 2017). However, there are few studies on delayed leaf senescence, especially in rice (Ren et al., 2007; Hörtensteiner, 2009; Morita et al., 2009). In this study, we found that salT could slow down the degradation of chlorophyll by reducing the expression of SAGs and CDGs, which could delay the senescence of leaves (Figures 1 and 2).

During leaf senescence, the most striking phenotypic change is the yellowing of the leaf caused by the preferential breakdown of chlorophyll and chloroplasts (Hilditch et al., 1989; Woo et al., 2001). The change in leaf color and chlorophyll content is integrally related to leaf senescence and is widely used in the quantification of senescence. CDRGs (OsSGR, OsNYC1, OsNYC3, and OsRCCR1) play important roles in regulating chlorophyll degradation, and the transcription levels of these genes are up-regulated during natural and dark-induced leaf senescence (Jiang et al., 2007; Park et al., 2007; Sato et al., 2009; Tang et al., 2011; Rong et al., 2013; Liu et al., 2016). The sgr mutant maintains greenness during rice leaf senescence, while overexpressing $S G R$ in rice produces yellowish-brown leaves (Park et al., 2007). The SGR gene encodes a chloroplast protein and is essential for the initiation of chlorophyll breakdown in plants (Park et al., 2007; Hörtensteiner, 2009; Liu et al., 2016).

The rice leaf nycl mutant also stays green during senescence, because chlorophyll degradation is impaired in the nycl mutant (Kusaba et al., 2007), and NYC1 is suggested to play essential roles in the regulation of LHCII and thylakoid membrane degradation during senescence (Kusaba et al., 2007). OsNYC3 encodes a plastid-targeted $\alpha / \beta$ hydrolase-fold family protein with an esterase/lipase motif, which affects chloroplast structure (Morita et al., 2009). OsRCCR1 encodes a red chlorophyll catabolite reductase, which plays a key role in the chlorophyll degradation pathway. The transcription level of OSRCCR1 is much lower in young leaves, but is about 20-fold higher in senescent leaves (Tang et al., 2011). These evidences suggest that OsSGR, OsNYC1, OsNYC3, and OsRCCR1 play important roles in regulating chlorophyll degradation during leaf senescence.

In this study, we found that the chlorophyll degradation rate of salT-OE plant leaves was lower than that of wild type during the mature period of rice (Figure 1E). Our results sbowed that the transcription levels of OsSGR, OsNYC1, OsNYC3, and OsRCCR1 were down-regulated during rice leaf senescence (Figure $3 \mathrm{~B}$ ). In addition, disadvantageous environmental factors, such as darkness, can also trigger senescence during leaf development (Zhang and Zhou, 2013). The results in Figure 4A showed that darkness can induce senescence in wild type, while overexpression of salT could delay leaf senescence in detached leaves. Moreover, the chlorophyll degradation rate of salT-OE plant leaves is lower than that of wild type during dark incubation (Figure 4B). The expression of $O s S G R$ and OsRCCR 1 was also inhibited with darkness treatment (Figure $4 C$ ). Our results showed that the leaves of salT-OE plants can stay green, because overexpression of the salT gene could inhibit the expression of CDGs (OSSGR, OsNYC1, OsNYC3, and OsRCCR1), which inhibits chlorophyll degradation during senescence in rice.

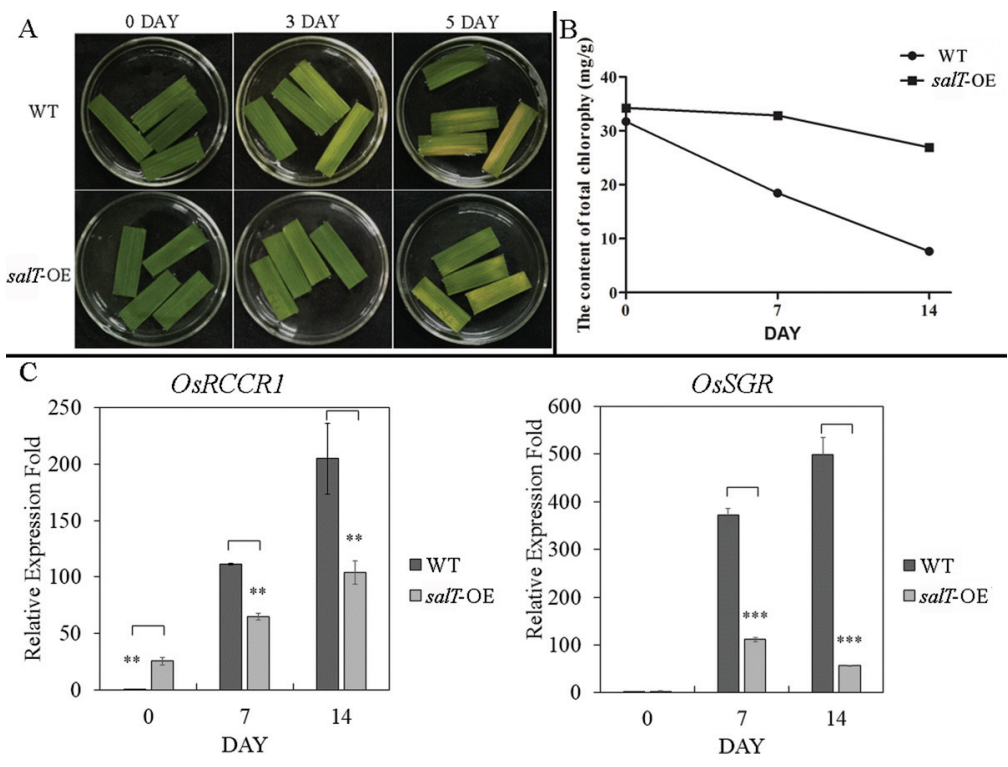

Figure 4 - Changes in the chlorophyll content of wild type leaves and of salT-OE lines during dark-induced senescence. (A) Changes in leaf color in detached leaves stored in darkness. (B) Chlorophyll contents and (C) relative transcript levels of chlorophyll degradation-related genes (OsSGR and OsRCCR1) of wild-type and salT-OE plants cultivated in darkness. Transcript levels are expressed relative to rice Actin1 in each sample, and values are reported as mean $\pm \mathrm{SD}(\mathrm{n}=3) .{ }^{*} p<0.05,{ }^{*} p<0.01,{ }^{*} p<0.001$. Student's t-test was used to generate $p$-value. 
Previous studies showed that some genes, such as SAGs, are up-regulated in senescent leaves. For example, the expression of AtNAP increases with aging; overexpression of AtNAP in wild type triggers precocious senescence and significantly blocks the function of the transcription factor that delays senescence (Guo and Gan, 2006). OsCATB is significantly induced by ABA (Abscicic Acid) (Agrawal et al., 2001; Ye et al., 2011). As a matter of fact, the up-regulation of SAGs is one of the hallmarks of leaf senescence (Kajimura et al., 2010). The results in Figure 3A show that the expression of SAGs (OSNAP and OSCATB) was not different from the wild type before leaf senescence, but was down-regulated during rice leaf senescence. This result indicates that the overexpression of salT could inhibit the expression of SAG gene and inhibit leaf senescence.

In general, overexpression of the salT gene could delay leaf senescence in rice by inhibiting the expression of SAGs and CDGs. Thus, salT is a new anti-aging factor gene, but its mechanism is still unclear and needs to be further explored.

\section{Acknowledgments}

This work was financially supported by the National Natural Science Foundation of China (No. 31671720 and No. 31201189), the Universities Natural Science Research Project of Jiangsu Province (12KJB210002), and the Distinguished Scholars Research Foundation of Jiangsu University (No. 10JDG134).

\section{Conflict of Interest}

The authors declare no conflict of interests.

\section{Author Contributions}

$\mathrm{KZ}$ and HT performed most of the experiments and wrote the manuscript; SX helped with the Realtime PCR analysis of genes; KL and WC helped with the transcriptome sequencing analysis; SZ and YY edited the article. All the authors discussed the results and contributed to the manuscript.

\section{References}

Agrawal GK, Rakwal R and Jwa NS (2001) Stress signaling molecules involved in defense and protein phosphatase 2A inhibitors modulate OsCATC expression in rice (Oryza sativa) seedlings. J Plant Physiol 158:1349-1355.

Buchanan-Wollaston V, Earl S, Harrison E, Mathas E, Navabpour S, Page T and Pink D (2003) The molecular analysis of leaf senescence-a genomics approach. Plant Biotechnol J 1:322.

Cao J, Jiang F, Sodmergen and Cui K (2003) Time-course of programmed cell death during leaf senescence in Eucommia ulmoides. J Plant Res 116:7-12.

Deng L, Qin P, Liu Z, Wang G, Chen W, Tong J, Xiao L, Tu B, Sun Y, Yan W et al. (2017) Characterization and finemapping of a novel premature leaf senescence mutant yel- low leaf and dwarf 1 in rice. Plant Physiol Biochem 111:50-58.

Gregersen PL and Holm PB (2007) Transcriptome analysis of senescence in the flag leaf of wheat (Triticum aestivum L.). Plant Biotech J 5:192-206.

Guo YF and Gan SS (2006) AtNAP, a NAC family transcription factor, has an important role in leaf senescence. Plant $\mathrm{J}$ 46:601-612.

Hayashi G, Moro CF, Rohila JS, Shibato J, Kubo A, Imanaka T, Kimura S, Ozawa S, Fukutani S, Endo S et al. (2015) 2D-DIGE-based proteome expression changes in leaves of rice seedlings exposed to low-level gamma radiation at Iitate village, Fukushima. Plant Signal Behav 10:e1103406.

He HG, Zhu SY, Jiang ZN, Ji YY, Wang F, Zhao RH and Bie TD (2016) Comparative mapping of powdery mildew resistance gene $P m 21$ and functional characterization of resistancerelated genes in wheat. Theor Appl Genet 4:819-829.

Hiei Y, Ohta S, Komari T and Kumashiro T (1994) Efficient transformation of rice (Oryza sativa L.) mediated by Agrobacterium and sequence analysis of the boundaries of the T-DNA. Plant J 6:271-282.

Hilditch PI, Thomas H, Thomas BJ and Rogers LJ (1989) Leaf senescence in a non-yellowing mutant of Festuca pratensis: Proteins of photosystem II. Planta 17:265-272.

Hortensteiner S (2009) Stay-green regulates chlorophyll and chlorophyll-binding protein degradation during senescence. Trends Plant Sci 14:155-162.

Jeon JS, Lee S, Jung KH, Jun SH, Jeong DH, Lee J, Kim C, Jang S, Yang K, Nam J et al. (2000) T-DNA insertional mutagenesis for functional genomics in rice. Plant J 22:561-570.

Jiang H, Li M, Liang N, Yan H, Wei Y, Xu X, Liu J, Xu Z, Chen F and $\mathrm{Wu} \mathrm{G}$ (2007) Molecular cloning and function analysis of the stay green gene in rice. Plant J 52:197-209.

John I, Hackett R, Cooper W, Drake R, Farrell A and Grierson D (1997) Cloning and characterization of tomato leaf senescence-related cDNAs. Plant Mol Biol 33:641-651.

Kajimura T, Mizuno N and Takumi S (2010) Utility of leaf senescence-associated gene homologs as developmental markers in common wheat. Plant Physiol Biochem 48:851-859.

Kusaba M, Ito H, Morita R, Iida S, Sato Y, Fujimoto M, Kawasaki S, Tanaka R, Hirochika H and Nishimura M (2007) Rice NON-YELLOW COLORING1 is involved in light-harvesting complex II and grana degradation during leaf senescence. Plant Cell 19:1362-1375.

Lee RH, Wang CH, Huang LT and Chen SC (2001) Leaf senescence in rice plants: Cloning and characterization of senescence up-regulated genes. J Exp Bot 52: 1117-1121.

Liang C, Wang Y, Zhu Y, Tang J, Hu B, Liu L, Ou S, Wu H, Sun X and Chu J (2014) OsNAP connects abscisic acid and leaf senescence by fine-tuning abscisic acid biosynthesis and directly targeting senescence-associated genes in rice. Proc Natl Acad Sci U S A 111:10013-10018.

Liu L, Zhou Y, Zhou G, Ye R, Zhao L, Li X and Lin Y (2008) Identification of early senescence-associated genes in rice flag leaves. Plant Mol Biol 67:37-55.

Liu L, Li HX, Zeng HL, Cai QS, Zhou X and Yin CX (2016) Exogenous jasmonic acid and cytokinin antagonistically regulate rice flag leaf senescence by mediating chlorophyll degradation, membrane deterioration, and senescence-associated genes expression. J Plant Growth Regul 35:366-376. 
Long SP, Zhu XG, Naidu SL and Ort DR (2006) Can improvement in photosynthesis increase crop yields? Plant Cell Environ 29:315-330.

Lu DZ, Fan YC, Ma YF, Lin ZD, Bao WQ, Jing YM and You SP (1988) Physiological and biochemical studies on leaf senescence at heading and grain formation stage in hybrid rice. Zhongguo Nongye Kexue, Sci Agric Sinica 3:21-26.

Masclaux-Daubresse C and Chardon F (2011) Exploring nitrogen remobilization for seed filling using natural variation in Arabidopsis thaliana. J Exp Bot 62:2131-2142.

Morita R, Sato Y, Masuda Y, Nishimura M and Kusaba M (2009) Defect in non-yellow coloring 3, an alpha/beta hydrolasefold family protein, causes a stay-green phenotype during leaf senescence in rice. Plant J 59:940-952.

Park SY, Yu JW, Park JS, Li J, Yoo SC, Lee NY, Lee SK, Jeong SW, Seo HS, Koh HJ et al. (2007) The senescence-induced staygreen protein regulates chlorophyll degradation. Plant Cell 19:1649-1664.

Raines T, Shanks C, Cheng CY, McPherson D, Argueso CT, Kim HJ, Franco-Zorrilla JM, Lopez-Vidriero I, Solano R, Vankova $\mathrm{R}$ et al. (2016) The cytokinin response factors modulate root and shoot growth and promote leaf senescence in Arabidopsis. Plant J 85:134-147.

Ren G, An K, Liao Y, Zhou X, Cao Y, Zhao H, Ge X and Kuai B (2007) Identification of a novel chloroplast protein AtNYE1 regulating chlorophyll degradation during leaf senescence in Arabidopsis. Plant Physiol 144:1429-1441.

Richards RA (2000) Selectable traits to increase crop photosynthesis and yield of grain crops. J Exp Bot 51:447-458.

Rong H, Tang Y, Zhang H, Wu P, Chen Y, Li M, Wu G and Jiang H (2013) The Stay-Green Rice like (SGRL) gene regulates chlorophyll degradation in rice. J Plant Physiol 170:13671373.

Sakuraba Y, Han SH, Yang HJ, Piao W and Paek NC (2016) Mutation of Rice Early Flowering3.1 (OsELF3.1) delays leaf senescence in rice. Plant Mol Biol 92:223-234.

Sato Y, Morita R, Katsuma S, Nishimura M, Tanaka A and Kusaba M (2009) Two short-chain dehydrogenase/reductases, NON-YELLOW COLORING 1 and NYC1-LIKE, are required for chlorophyll $\mathrm{b}$ and light-harvesting complex II degradation during senescence in rice. Plant J 57:120-131.

Schippers JH (2015) Transcriptional networks in leaf senescence. Curr Opin Plant Biol 27:77-83.

Tan XL, Huang Q, Tan RK, Wu L, Zhang ZY, Wang Z, Lu CM and Li XF (2015) Cloning and Functional Characterization of a Fatty Acyl-Acyl Carrier Protein Thioesterase Gene
(BnFatB) in Brassica napus L. J Agric Sci Technol 4:987997.

Tang Y, Li M, Chen Y, Wu P, Wu G and Jiang H (2011) Knockdown of $O s P A O$ and $O s R C C R 1$ cause different plant death phenotypes in rice. J Plant Physiol 168:1952-1959.

Thomas H and Howarth CJ (2000) Five ways to stay green. J Exp Bot 51:329-337.

Watanabe A and Imaseki H (1982) Changes in translatable mRNA in senescing wheat leaves. Plant Cell Physiol 23:489-497.

Woo HR, Chung KM, Park JH, Oh SA, Ahn T, Hong SH, Jang SK and Nam HG (2001) ORE9, an F-box protein that regulates leaf senescence in Arabidopsis. Plant Cell 13:1779-1790.

Wu XY, Hu WJ, Luo H, Xia Y, Zhao Y, Wang LD, Zhang LM, Luo JC and Jing HC (2016) Transcriptome profiling of developmental leaf senescence in sorghum (Sorghum bicolor). Plant Mol Biol 92:555-580.

Yang J and Zhang J (2006) Grain filling of cereals under soil drying. New Phytol 169:223-236.

Ye NH, Zhu GH, Liu YG, Li YX and Zhang JH (2011) ABA controls $\mathrm{H}_{2} \mathrm{O}_{2}$ accumulation through the induction of OsCATB in rice leaves under water stress. Plant Cell Physiol 52:689698.

Zhang H and Zhou C (2013) Signal transduction in leaf senescence. Plant Mol Biol 82:539-545.

Zhao CC, Huang FD, Gong P, Yang X, Cheng FM and Pan G (2014) Physiological characteristics and gene mapping of a leaf early-senescence mutant osled in rice. Acta Agron Sinica 40:1946-1955.

Zhu K, Min C, Xia H, Yang Y, Wang B and Chen K (2014) Characterisation of Indica Special Protein (ISP), a marker protein for the differentiation of Oryza sativa subspecies indica and japonica. Int J Mol Sci 15:7332-7343.

Zuo J and Li J (2014) Molecular genetic dissection of quantitative trait loci regulating rice grain size. Annu Rev Genet 48:99118.

\section{Supplementary material}

The following online material is available for this article: Table S1 - Primers used in this study.

Associate Editor: Marcia Pinheiro Margis

License information: This is an open-access article distributed under the terms of the Creative Commons Attribution License (type CC-BY), which permits unrestricted use, distribution and reproduction in any medium, provided the original article is properly cited. 